\title{
Minimally Invasive Circumferential Hiatal Dissection for the Treatment of Adenocarcinoma of the Distal Esophagus and Esophago-gastric Junction: Technical Considerations Combined With Histopathological Outcomes
}

\author{
BRUNO LORENZI ${ }^{1}$, SPYRIDON DAVAKIS ${ }^{1}$, ATHANASIOS SYLLAIOS ${ }^{1}$, ALI KORDZADEH ${ }^{1}$, MOINA KADRI $^{2}$, \\ MANISHA RAM ${ }^{2}$, KHALEEL FAREED ${ }^{3}$, CHARLOTTE BARTER $^{3}$ and ALEXANDROS CHARALABOPOULOS ${ }^{1}$ \\ ${ }^{1}$ Regional Center for Oesophagogastric Cancer, Department of Upper Gastrointestinal Surgery, \\ Mid Essex Hospital Services NHS Trust, Broomfield Hospital, Chelmsford, U.K.; \\ ${ }^{2}$ Department of Pathology, Mid Essex Hospital Services NHS Trust, Broomfield Hospital, Chelmsford, U.K.; \\ ${ }^{3}$ Department of Surgery, Derby Teaching Hospitals NHS Foundation Trust, Royal Derby Hospital, Derby, U.K.
}

\begin{abstract}
Background/Aim: Circumferential resection margin involvement is an independent prognostic factor in patients with adenocarcinoma of the distal esophagus and esophagogastric junction. However, there is currently no consensus on the extent and the technique of hiatal dissection. We describe a minimally invasive technique of circumferential hiatal dissection for adenocarcinoma of the distal esophagus and esophago-gastric junction with its related histopathological results. Patients and Methods: A prospective study of 40 consecutive patients undergoing hybrid (laparoscopic/ thoracotomic) or totally minimally invasive Ivor-Lewis esophagogastrectomy over a period of 21 months was conducted. Dissection of the hiatus included peri-esophageal surrounding tissues in a cylindrical fashion maximizing the distance from the oesophageal wall. Crural muscle fibers and pleura bilaterally, pericardial fat anteriorly and pre-aortic tissue posteriorly were excised en bloc. Histopathological results particularly focused on involvement of the circumferential resection margin. Neoadjuvant chemotherapy was given to 24 (60\%) patients. Results: Complete histological clearance $(R 0)$ was achieved in $92.5 \%(n=37)$ according to the criteria of the College of American Pathologists and in $87.5 \%$ $(n=35)$ according to those of the Royal College of Pathologists.
\end{abstract}

Correspondence to: Spyridon Davakis, MD, General Surgery Registrar, Regional Center for Oesophagogastric Cancer, Department of Upper Gastrointestinal Surgery, Mid Essex Hospital Services NHS Trust, Broomfield Hospital, Court Rd, Chelmsford, CM1 7ET, Essex, U.K. Tel: +44 7553104746, Mobile: +30 6973762451,e-mail: spdavakis@gmail.com

Key Words: Esophageal carcinoma, esophagectomy, circumferential resection margin.
In pT3 tumors $(n=22)$, the circumferential resection margin was negative in 20 patients (91\%) according to the College of American Pathologists, and in 17 (77\%) according to the Royal College of Pathologists. Conclusion: Adoption of this safe and reproducible technique might reduce the incidence of circumferential resection margin involvement and improve pathological outcomes. In addition, there may be positive implications for training and quality control.

Minimally invasive esophagectomy is being increasingly used in the treatment of adenocarcinoma of the distal esophagus (DE) and esophago-gastric junction (EGJ). However, there is currently no consensus on the extent and the technique of hiatal dissection, despite two recent meta-analyses having shown that involvement of the circumferential resection margin (CRM) is an independent prognostic factor in patients with operable esophageal cancer $(1,2)$. Furthermore, achieving resection clearance has been proposed as an indicator of surgical quality in esophagectomy, and is indeed the only oncological factor that can be influenced by the surgeon (3-5).

Despite the well-known biological differences between rectal and distal esophageal adenocarcinoma, similarities have been described in surgical technique with comparable oncological implications (6). In the past two decades, the introduction and standardization of total mesorectal excision (TME) has been associated with reduction of CRM involvement, as well as lower incidence of local recurrence in patients with rectal cancer (7). A laparoscopic approach in the treatment of rectal cancer has been adopted by many surgeons, and a consensus on surgical technique has been universally accepted (8). A similar process is currently evolving with the introduction of extralevator abdominoperineal excision (ELAPE), which seems to be oncologically superior to the standard abdominoperineal resection $(9,10)$. 
The aim of this study was to describe our minimally invasive technique of circumferential hiatal dissection (CHD) for the treatment of adenocarcinoma of the DE and EGJ and its related histopathological results, with particular focus on CRM involvement. Our technique involves precise and meticulous dissection of the hiatus by maintaining a standard maximum distance from the esophageal wall and the tumour in a cylindrical fashion, following the TME and ELAPE principles of rectal cancer surgery.

\section{Patients and Methods}

A prospective study of 40 consecutive patients treated for adenocarcinoma of the DE and EGJ (Siewert type I and II only) that underwent either hybrid (laparoscopic/thoracotomic) or totally minimally invasive 2 -stage esophagogastrectomy was conducted. Operations were performed by an experienced consultant surgeon (BL or AC) from March 2015 to January 2017. The study was approved by the Institutional Review Board (number: ca18-132).

Staging was based on clinical assessment, upper gastrointestinal endoscopy with biopsies, computed tomography of the chest and abdomen, and endoscopic ultrasound. Five (12.5\%) patients did not undergo endoscopic ultrasound because of technical reasons (esophageal stent) or patient choice. Computed tomographypositron-emission tomography and staging laparoscopy were also performed in all cases. Treatment strategy was always discussed and agreed in a multidisciplinary team setting. The response to neoadjuvant chemotherapy was evaluated according to the RECIST criteria (11). The study end-point was set at 30- and 90-day mortality. Postoperative complications were recorded according to the criteria set by Low et al. (12).

Patient position. The patient was placed in supine position with their arms by their side and legs split and secured on vertical supports. Straps were used to achieve two-point fixation above and below the knees. A steep reverse Trendelenburg position $\left(>30^{\circ}\right)$ was utilized for optimal hiatus exposure. The operating surgeon stood between the legs with the first assistant on the patient's left and the camera holder on the right.

Port position. A total of four abdominal ports were used. Our preference was to enter the abdominal cavity using a $12 \mathrm{~mm}$ bladeless optical viewing trocar loaded with a $10 \mathrm{~mm}$ laparoscope in the left upper quadrant (subcostal region). Pneumoperitoneum was obtained by insufflation of the abdominal cavity with $\mathrm{CO}_{2}$ at a pressure of $12 \mathrm{mmHg}$. The rest of the ports were inserted under direct vision: two $12 \mathrm{~mm}$ ports in the right upper quadrant and epigastrium, and a $5 \mathrm{~mm}$ port in the left flank (anterior axillary line). A further $5 \mathrm{~mm}$ subxiphoid incision was made to facilitate the insertion of a Nathanson's retractor for retraction of the left liver. A $30^{\circ}$ laparoscope was used for optimal viewing.

Anatomical landmarks and operative technique. Once gastric mobilization and upper abdominal lymph node dissection was performed, the hiatal dissection was initiated starting from the right crus. The incision was performed leaving a cuff of crural muscle fibers attached to the specimen (Figure 1A). The dissection was continued cranially in a slightly diagonal direction (following the direction of the esophagus) ensuring that crural fibers stayed with the esophagus and the tumor along the entire esophageal length until the right pleura was opened. Similarly, the left crus was identified and the incision was performed through the muscle leaving a cuff of crural fibers on the specimen (Figure 1B). The dissection continued until the left pleura was opened. The pericardium was the anatomical landmark during the anterior dissection (Figure 1C). A vertical $1 \mathrm{~cm}$ 'slit' on the central tendon of the diaphragm was performed for better exposure of the pericardial fat. Anteriorly, the pericardial fat was excised en bloc with the specimen, leaving the pericardium exposed. Posteriorly, the landmark was the descending thoracic aorta (Figure 1D). The posterior crural fibers were divided and care was taken to continue the dissection along the plane of nodal dissection at the level of the coeliac axis until the aorta was visualized. The pre-aortic fascia was incised and resected en bloc with the specimen. The dissection was carried on cranially for approximately $5 \mathrm{~cm}$.

The end result was an en bloc resection of the DE and EGJ together with the muscle fibers of the crura, as well as the lower mediastinal pleura bilaterally, the pericardial fat anteriorly and the pre-aortic tissue posteriorly (Figure 2).

Anesthetic considerations. When both pleural cavities were entered, mild tachycardia, hypotension and high airway pressure mimicking a condition similar to tension pneumothorax was occasionally observed. This was a consequence of $\mathrm{CO}_{2}$ moving into the right and left pleural cavities. All patients tolerated these physiological changes well, but adjustments to ventilation settings were necessary. In order to minimize these effects, before entering the pleural space, the $\mathrm{CO}_{2}$ insufflation pressure was reduced from 12 to $8 \mathrm{~mm} \mathrm{Hg}$ and this step of the procedure was performed at the very end of the abdominal phase of the operation.

Histopathology. The specimen was fixed for at least 24 hours in $10 \%$ buffered formalin solution. Prior to fixation, the outer surfaces of the specimen were inked to accurately assess the CRM clearance microscopically. Serial cross-sectional slicing was performed at 5 to $10 \mathrm{~mm}$ intervals. Cross-sections were performed by a consultant histopathologist specialized in upper gastrointestinal malignancies. Macroscopic items reported included specimen type with dimensions, tumor location, diameter, morphology, anatomical tumor depth and distance from circumferential and longitudinal margins. Microscopic items reported were tumor type, differentiation and size, clearance from margins, presence of lymphovascular and perineural invasion, lymph node status, and Mandard regression score when applicable. The Mandard score ranged from 1 (complete regression with no evidence of residual viable malignant cells) to 5 (no regression) (13). Pathological staging was reported according to the seventh edition of the American Joint Committee on Cancer TNM system (14).

Currently, there is discrepancy between the definition of surgical clearance and CRM involvement in esophageal cancer provided by the College of American Pathologists (CAP) (15) and the Royal College of Pathologists (RCP) (16). Therefore, the CRM status was reported according to both $\mathrm{CAP}$ and RCP criteria.

Statistical analysis. All continuous variables are reported as medians with their corresponding ranges and percentages. Statistical analysis was performed using SPSS software version 20.0 (IBM, Armonk, NY, USA). 

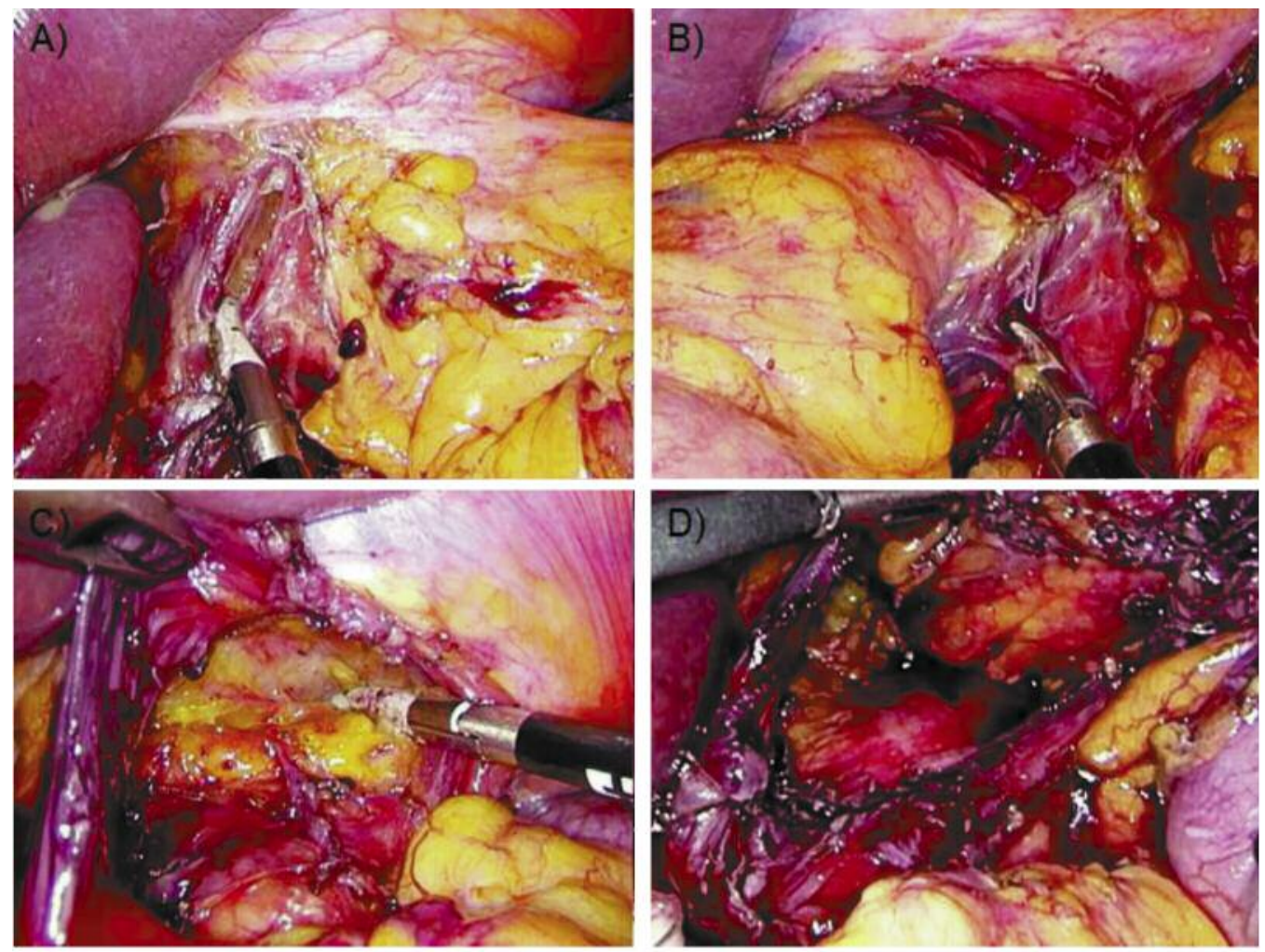

Figure 1. Operative steps. A: Lateral dissection at the level of the right crus. B: lateral dissection at the level of the left crus. C: Anterior dissection and pericardium. D: Posterior dissection and descending thoracic aorta.
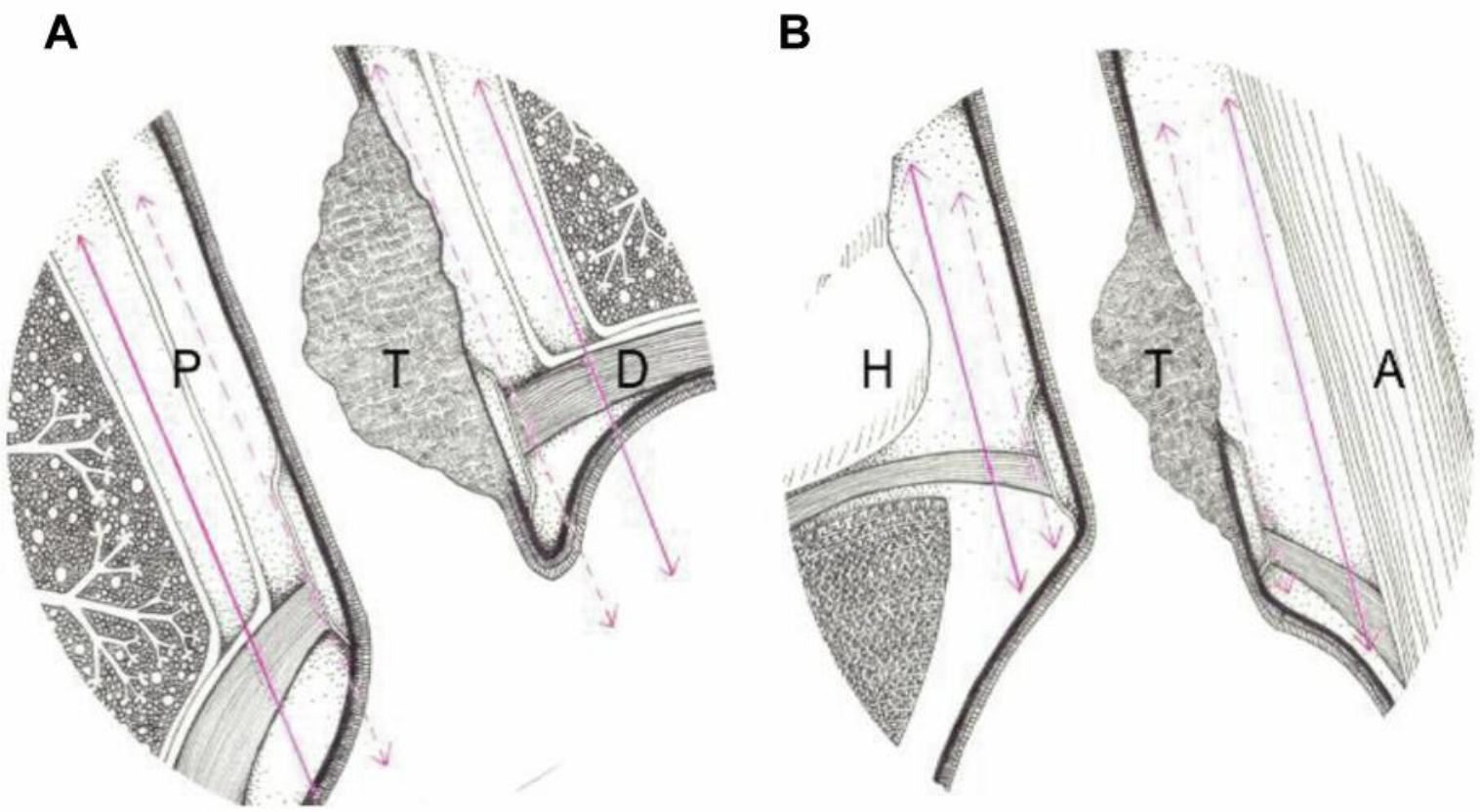

Figure 2. Illustrations demonstrating the cylindrical resection of the hiatus during circumferential hiatal dissection (straight line) in comparison to a less radical peri-esophageal dissection (dotted line). A: Coronal view. B: Sagittal view. P: Pleura; T: tumour; D: diaphragm; H: heart; A: aorta. 


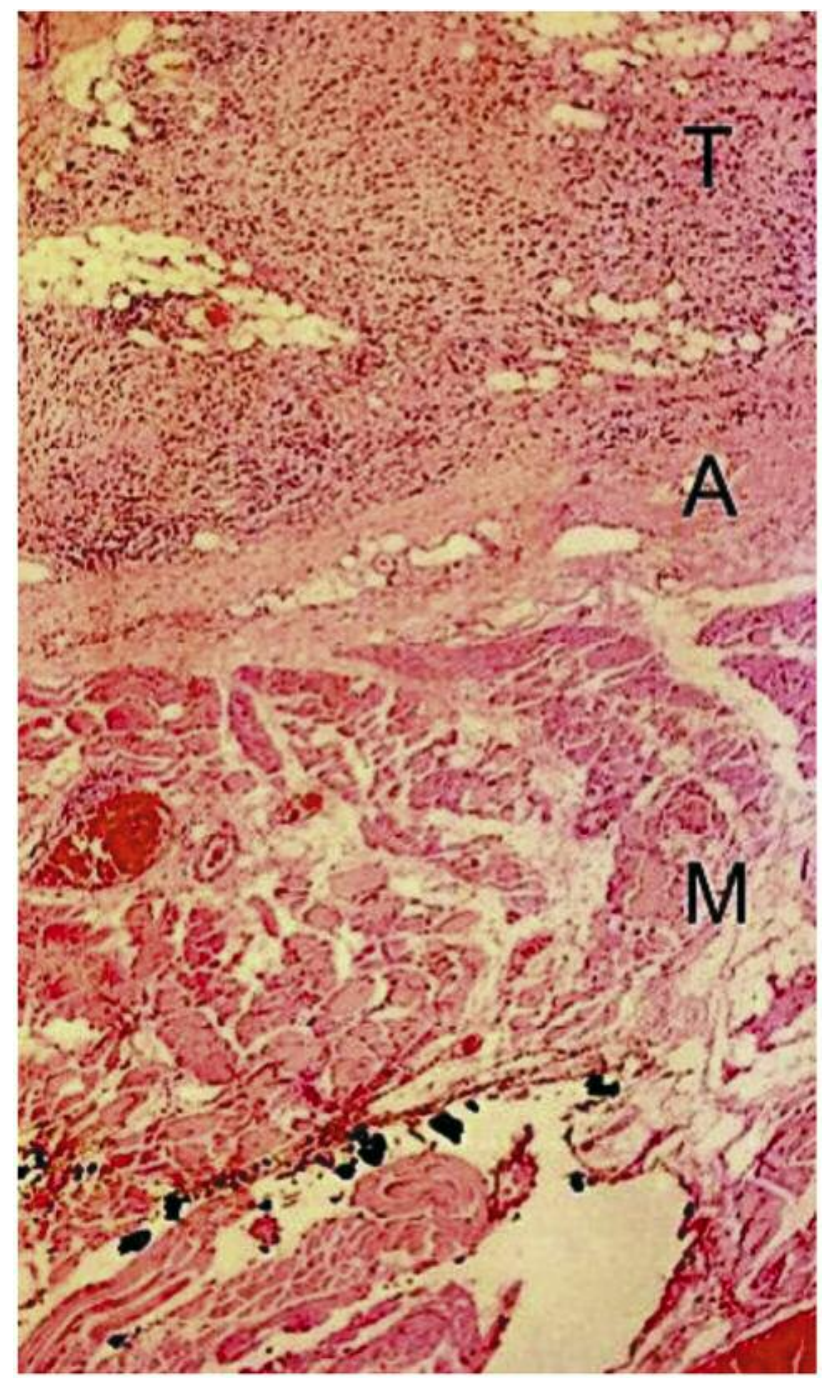

Figure 3. Histopathological image showing the circumferential resection margin at the level of the esophagogastric junction in a patient with pT3 tumor after laparoscopic circumferential hiatal dissection. R0 resection was achieved because of inclusion of fibers of the diaphragm (skeletal muscle). T: Tumor; A: adventitia; M: muscle. Line $0.5 \mathrm{~mm}$.

\section{Results}

The study cohort included 40 patients. Their median age was 62.5 years (range $=31-82$ years) with male predominance [male $\mathrm{n}=37 \quad(92.5 \%) \quad$ vs. female $\mathrm{n}=3 \quad(7.5 \%)]$. Neoadjuvant chemotherapy was given to 24 patients $(60 \%)$. The preoperative chemotherapy regimen was a combination of intravenous epirubicine and cisplatin with oral capecitabine (ECX) in 18 patients $(75 \%)$ and intravenous epirubicin/ oxaliplatin with oral capecitabine (EOX) in six patients $(25 \%)$. Of the patients who had neoadjuvant chemotherapy, 17 (71\%) completed all three cycles. One patient (4\%) had an extended course of neoadjuvant chemotherapy (three cycles of EOX plus four cycles of oxaliplatin). The remaining six patients (25\%) had a reduced dose of chemotherapy for various reasons (age, side-effects or complications). According to RECIST criteria, partial response was observed in 15 patients (63\%), stable disease in eight $(33 \%)$ and progressive disease (without evidence of distant metastasis) in one (4\%).

Operative approach was hybrid (laparoscopic/thoracotomic) in 31 patients $(77 \%)$, and totally minimally invasive Ivor-Lewis esophagogastrectomy in nine cases (23\%). Twenty-three patients $(57 \%)$ had feeding jejunostomy inserted intraoperatively. Of the 24 patients who received neoadjuvant chemotherapy, nine (37\%) had postoperative treatment (chemotherapy for eight and radiotherapy for one). One patient who had primary surgery had adjuvant chemotherapy.

We encountered three $(7.5 \%)$ anastomotic leaks (two type I and one type II), three (7.5\%) chyle leaks (two type IA and one type IIA), one (2.5\%) vocal cord palsy (type IA), two $(5 \%)$ hiatal hernias, six $(15 \%)$ cases of pneumonia and one $(2.5 \%)$ of pleural effusion requiring drainage. Thirty-day mortality was $2.5 \%$ and 90 -day mortality was $10 \%$. For overall mortality, one patient died on the 22 nd postoperative day due to respiratory failure and pneumonia, one patient developed a broncho-esophageal fistula and died on the 34th postoperative day, and three patients died of progressive metastatic disease following discharge.

Eleven $(27.5 \%)$ patients had adenocarcinoma of the DE and $29(72.5 \%)$ had adenocarcimoma of the EGJ. Of the latter group, $10(25 \%)$ were of Siewert type I, and the remaining 19 $(47.5 \%)$ were of Siewert type II. The grade of differentiation was well-differentiated in three cases $(7.5 \%)$, moderately differentiated in $13(32.5 \%)$, and poorly differentiated in 24 (60\%). Tumor was pT0 in two cases $(5 \%)$, pTis in two $(5 \%)$, pT1a in two $(5 \%)$, pT1b in four $(10 \%)$, pT2 in eight $(20 \%)$, and pT3 in $22(55 \%)$. pN0 was in 18 patients $(45 \%)$, pN1 in $11(27.5 \%), \mathrm{pN} 2$ in four $(10 \%)$ and $\mathrm{pN} 3$ in seven $(17.5 \%)$. The median number of lymph nodes removed was 29 (range $=11-60$ ). Lymphovascular and perineural invasion was present in $18(45 \%)$ and $15(37.5 \%)$ cases, respectively.

R0 was achieved in $\mathrm{n}=37$ patients $(92.5 \%)$ according to CAP, and in $\mathrm{n}=35(87.5 \%)$ according to RCP. CRM was negative in 38 patients (95\%) according to CAP, and in 35 (87.5\%) according to RCP. Of the 22 patients (55\%) with pT3 tumours treated with CHD in this series, CRM was negative in 20 patients $(91 \%)$ according to CAP, and in $17(77 \%)$ according to RCP. The median CRM of patients was $3 \mathrm{~mm}$ (range $=0-15 \mathrm{~mm}$ ). The median CRM of pT3 tumours was 1.4 $\mathrm{mm}$ (range $=0-7.5 \mathrm{~mm}$ ). The CRM coincided with the EGJ in 12 patients $(30 \%)$. Of the 24 patients who received neoadjuvant chemotherapy, four $(17 \%)$ had a Mandard score of 1 , two of $3(9 \%)$, nine of $4(37 \%)$ and nine of $5(37 \%)$. The complete details of the final histopathological results are shown in Table I. 


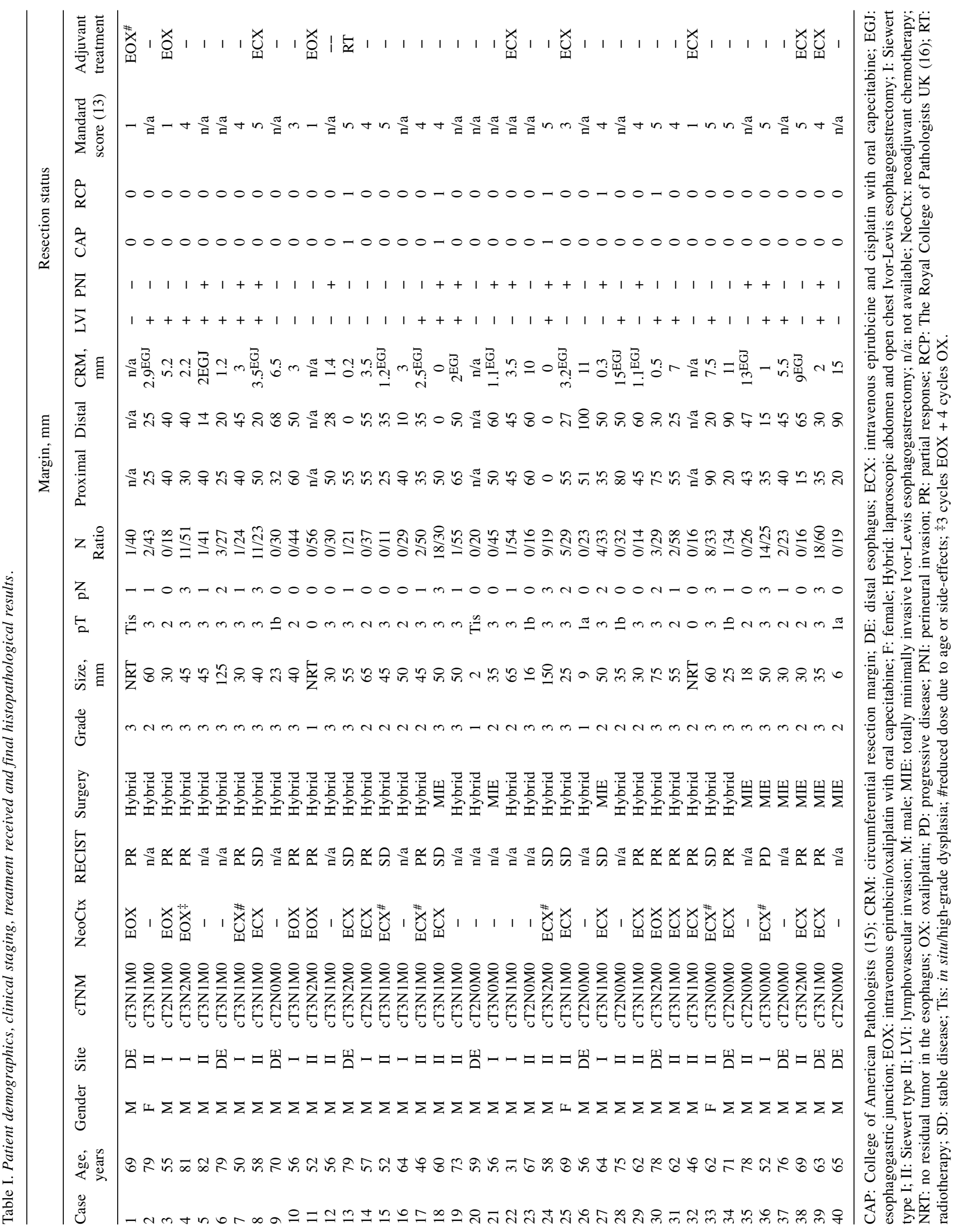




\section{Discussion}

The adoption of the described surgical technique (CHD) for resection of adenocarcinoma of the distal esophagus and EGJ led to encouraging pathological outcomes in our experience. Complete clearance (R0) was achieved in $92.5 \%$ of individuals according to CAP, and $87.5 \%$ according to RCP. In addition, CRM involvement in pT3 tumors was only $9 \%$ according to CAP and $23 \%$ to RCP. Our results compare favorably with the rates of CRM involvement reported in recent meta-analyses. In the study of Wu et al., the median positive rates of CRM were $17 \%$ and $40 \%$ according to CAP and RCP, respectively (2). Chan et al. described corresponding values of $15 \%$ and $43 \%$ for pT3 tumors (1).

In the attempt to define the optimal surgical technique for tumors of the EGJ, Botha et al. described "total adventitial resection of the cardia" (TARC) (6). In their work, 40 patients with tumors of the EGJ underwent surgery within a period of 2 years, and had an en bloc resection of a cuff of hiatus, the pericardial fat pad, the lower mediastinal pleura and the preaortic adventitia. The series included 19 patients who underwent an Ivor-Lewis esophagogastrectomy, 16 who underwent trans-hiatal resections, four extended total gastrectomies and one left thoracotomy. The reported percentage of R0 resection according to RCP was $68 \%(n=27)$. Twelve patients had R1 resections due to positive CRM (eight with tumor at the circumferential margin, and four with tumor at $<1 \mathrm{~mm}$ ) and one because of a positive longitudinal margin.

Compared to Botha et al., we presented a more homogeneous series where all patients underwent a minimally invasive dissection of the hiatus as part of a two-stage IvorLewis esophagogastrectomy with 2-field lymph node dissection. In our view, this is a crucial technical point, and might explain the differences in the CRM results $(87.5 \% \mathrm{vs}$. $68 \%$ ) considering that half of the patients did not undergo radical mediastinal dissection following TARC. We observed that the CRM corresponded with the level of the EGJ in a minority of patients in our series $(n=12,30 \%)$ (Figure 3 ). However, laparoscopic CHD led to the natural planes of radical mediastinal dissection of the esophagus and mesoesophagus described by Cuesta et al. (17). We believe that the improvement of CRM in our series was a combined result of laparoscopic CHD and radical mediastinal dissection.

Another relevant difference is that we preferred to start the hiatal dissection from the right crus after excision of the pars flaccida of the gastro-hepatic ligament. This represents another potential explanation of the differences in CRM involvement. The anterior and posterior dissection of the hiatus is similar to TME for rectal tumor as a plane between the pericardium and the pericardial fat, as well as at the level of the pre-aortic fascia, can easily be created and followed. However, the right and left dissection is performed in a nonanatomical plane more in line with the principles of prone
ELAPE. If the initial approach of the hiatus is anterior, there is a potential risk of entering the pleura too early and possibly losing the cylindrical fashion of the dissection.

By removing a wider specimen at the hiatus, we acknowledge that we might create a larger defect and potentially face a higher risk of diaphragmatic hernia. Similarly, in rectal surgery, the closure of the perineal defect after ELAPE can require a mesh or a myocutaneous flap (9, 10). Nevertheless, our results compare well with the incidence of hiatus hernia reported in other series (18-21).

Patients with $\mathrm{T} 1$ and $\mathrm{T} 2$ disease should have $\mathrm{R} 0$ resection by definition (at least by CAP criteria) and it could be argued that less radical surgery would suffice. However, it is not simple to achieve correct staging prior to surgery (22). In our series, there were cases clinically overstaged, as well as understaged, compared to the final histopathological results. We routinely performed laparoscopic CHD for all our patients irrespective of the clinical stage in order to avoid unsatisfactory pathological outcomes. We also believe that a standardized surgical technique may have positive implications in training, assessment and quality control.

\section{Conclusion}

We described a simple, safe and reproducible minimally invasive technique of hiatal dissection for the treatment of DE and EGJ adenocarcinoma. Adoption of this surgical technique might improve pathological results by reducing the incidence of CRM involvement, as well as having positive implications in training and quality control. A limitation of this study is the lack of long-term follow-up, which will be required in future studies, in order to establish whether the improved CRM results would translate into a survival benefit.

\section{Conflicts of Interest}

None of the Authors declare any potential conflict of interest. No funding was received.

\section{Authors' Contributions}

Study conception and design: BL, SD, AS, AK, MK, MR, KF, CB, and AC. Acquisition of data: BL, AK, MK, MR, KF, CB, and AC. Analysis and interpretation of data: BL, AK, MK, MR, and AC. Drafting of article: BL, SD, AS, AK, MR, KF, CB, and AC. Critical revision: BL, SD, AS, AK, MK, MR, and AC. All Authors approved the final version of the article.

\section{Ethical Approval}

Informed consent was obtained from all the patients included in the current study. All procedures followed were in accordance with the ethical standards of human experimentation (institutional and national) and with the Helsinki declaration of 1964 and later versions. 


\section{References}

1 Chan DS, Reid TD, Howell I and Lewis WG: Systematic review and meta-analysis of the influence of circumferential resection margin involvement on survival in patients with operable oesophageal cancer. Br J Surg 100: 456-464, 2013. PMID: 23319427. DOI: $10.1002 /$ bjs. 9015

$2 \mathrm{Wu}$ J, Chen QX, Teng LS and Krasna MJ: Prognostic significance of positive circumferential resection margin in esophageal cancer: A systematic review and meta-analysis. Ann Thorac Surg 97: 446-453, 2014. PMID: 24365211. DOI: 10.1016/j.athoracsur.2013.10.043

3 Lerut T, Nafteux P, Moons J, Coosemans W, Decker G, De Leyn $\mathrm{P}$ and Van Raemdonck D: Quality in the surgical treatment of cancer of the esophagus and gastroesophageal junction. Eur $\mathbf{J}$ Surg Oncol 31: 587-594, 2005. PMID: 16023943. DOI: 10.1016/ j.ejso.2005.02.010

4 Izon AS, Jose P, Hayden JD and Grabsch HI: Significant variation of resected meso-esophageal tissue volume in twostage subtotal esophagectomy specimens: a retrospective morphometric study. Ann Surg Oncol 20: 788-797, 2013. PMID: 22983387. DOI: $10.1245 / \mathrm{s} 10434-012-2659-\mathrm{x}$

5 Crosby T, Evans M, Gillies RS and Maynard ND: The management of a patient with an operable carcinoma of the oesophagus. Ann R Coll Surg Engl 91: 366-370, 2009. PMID: 19622256. DOI: $10.1308 / 003588409 X 432428$

6 Botha AJ, Odendaal W, Patel V, Watcyn-Jones T, Mahadeva U, Chang $\mathrm{F}$ and Deere H: Total adventitial resection of the cardia: "optimal local resection" for tumours of the oesophagogastric junction. Ann R Coll Surg Eng 93: 608-614, 2011. PMID: 22041237. DOI: $10.1308 / 003588411 X 13165261994111$

7 Carlsen E, Schlichting E, Guldvog I, Johnson E and Heald RJ: Effect of the introduction of total mesorectal exicion for the treatment of rectal cancer. Br J Surg 85: 526-529, 1998. PMID: 9607540. DOI: 10.1046/j.1365-2168.1998.00601.x

8 Miskovic D, Foster J, Agha A, Delaney CP, Francis N, Hasegawa H, Karachun A, Kim SH, Law WL, Marks J, Morino M, Panis Y, Uriburu JC, Wexner SD and Parvaiz A: Standardization of laparoscopic total mesorectal excision for rectal cancer. A structured international expert consensus. Ann Surg 261: 716-722, 2015. PMID: 25072446. DOI: 10.1097/ SLA.0000000000000823

9 Butt HZ1, Salem MK, Vijaynagar B, Chaudhri S and Singh B: Perineal reconstruction after extra-levator abdominoperineal excision (eLAPE): A systematic review. Int J Colorectal Dis 28 : 1459-1468, 2013. PMID: 23440362. DOI: 10.1007/s00384-0131660-6

$10 \mathrm{Yu} \mathrm{HC1}$, Peng H, He XS and Zhao RS: Comparison of short- and long-term outcomes after extralevator abdominoperineal excision and standard abdominoperineal excision for rectal cancer: A systematic review and meta-analysis. Int J Colorectal Dis 29: 183191, 2014. PMID: 24271080. DOI: 10.1007/s00384-013-1793-7

11 Therasse P, Eisenhauer EA and Verweij J: RECIST revisited: A review of validation studies on tumour assessment. Eur J Cancer 42: 1031-1039, 2006. PMID: 16616487. DOI: 10.1016/ j.ejca. 2006.01.026

12 Low DE, Alderson D, Cecconello I, Chang AC, Darling GE, D'Journo XB, Griffin SM, Hölscher AH, Hofstetter WL, Jobe BA, Kitagawa Y, Kucharczuk JC, Law SY, Lerut TE, Maynard N, Pera M, Peters JH, Pramesh CS, Reynolds JV, Smithers BM and van Lanschot JJ: International Consensus on Standardization of Data Collection for Complications Associated With
Esophagectomy: Esophagectomy Complications Consensus Group (ECCG). Ann Surg 262: 286-294, 2015. PMID: 25607756. DOI: $10.1097 /$ SLA.0000000000001098

13 Mandard AM, Dalibard F, Mandard JC and Marnay J: HenryAmar M, Petiot JF, Roussel A, Jacob JH, Segol P, Samama G and Ollivier JM, Bonvalot $\mathrm{S}$ and Gignoux $\mathrm{M}$ : Pathologic assessment of tumor regression after pre-operative chemoradiotherapy of esophageal carcinoma. Clinicopathologic correlations. Cancer 73(11): 2680-2686, 1994. PMID: 8194005.

14 Rice TW, Blackstone EH and Rusch VW: Seventh Edition of the AJCC Cancer Staging Manual: Esophagus and Esophagogastric Junction. Ann Surg Oncol 17: 1721-1724, 2010. PMID: 20369299. DOI: 10.1245/s10434-010-1024-1

15 Washington K, Carter DK, Fritzgibbons P, Burgart LJ, Carter DK, Fitzgibbons P, Frankel WL, Jessup J, Kakar S, Minsky B, Nakhleh R and Laura H: Tang LH and Compton CC: Protocol for the examination of specimens from patients with carcinoma of the esophagus. College of American Pathologists, 2011. Available at http://www.cap.org/apps/docs/committees/ cancer/ cancer_protocols/2012/Esophagus_12protocol_3111.pdf. Accessed February 22, 2017.

16 The Royal College of Pathologists: Dataset for the histopathological reporting of oesophageal carcinoma, 2nd ed, 2007. Available at https://www.rcpath.org/resourceLibrary/g006 oesophagealdatasetfinalfeb07-pdf.html. Accessed February 22, 2017.

17 Cuesta MA, Weijs TJ, Bleys RL, van Hillegersberg R, van Berge Henegouwen MI, Gisbertz SS, Ruurda JP, Straatman J, Osugi H and van der Peet DL: A new concept of the anatomy of the thoracic oesophagus: The meso-oesophagus. Observational study during thoracoscopic esophagectomy. Surg Endosc 29: 2576-2582, 2015. PMID: 25480608. DOI: 10.1007/s00464-014-3972-1

18 Price TN, Allen MS, Nichols FC 3rd, Cassivi SD, Wigle DA, Shen KR and Deschamps C: Hiatal hernia after esophagectomy: analysis of 2,182 esophagectomies from a single institution. Ann Thorac 92(6): 2041-2045, 2011. PMID: 22115216. DOI: 10.1016/j.athoracsur.2011.08.013

19 Ganeshan DM, Correa AM, Bhosale P, Vaporciyan AA, Rice D, Mehran RJ, Walsh GL, Iyer R, Roth JA, Swisher SG and Hofstetter WL: Diaphragmatic hernia after esophagectomy in 440 patients with long-term follow-up. Ann Thorac Surg 96: 1138-1145, 2013. PMID: 23810174. DOI: 10.1016/j.athoracsur.2013.04.076

20 Benjamin G, Ashfaq A, Chang YH, Harold K and Jaroszewski D: Diaphragmatic hernia post-minimally invasive esophagectomy: A discussion and review of literature. Hernia 19: 635-643, 2015. PMID: 25739716. DOI: 10.1007/s10029-015-1363-8

21 Brenkman HJ, Parry K, Noble F, van Hillegersberg R, Sharland D, Goense L, Kelly J, Byrne JP, Underwood TJ and Ruurda JP: Hiatal hernia after esophagectomy for cancer. Ann Thorac Surg 103: 1055-1062, 2017. PMID: 28267979. DOI: 10.1016/ j.athoracsur.2017.01.026

22 Zhang JQ, Hooker CM, Brock MV, Shin J, Lee S, How R, Franco N, Prevas H, Hulbert A and Yang SC: Neoadjuvant chemoradiation therapy is beneficial for clinical stage T2 N0 esophageal cancer patients due to inaccurate preoperative staging. Ann Thorac Surg 93: 429-437, 2012. PMID: 22269708. DOI: 10.1016/j.athoracsur.2011.10.061

Received April 14, 2019

Revised May 4, 2019

Accepted May 8, 2019 